\title{
Alienación y satisfacción laboral en la industria de vestiduras automotrices: Caso Ciudad Juárez, Chihuahua
}

Galván Mendoza, Oscar; Esquinca Moreno, Armando

Alienación y satisfacción laboral en la industria de vestiduras automotrices: Caso Ciudad Juárez, Chihuahua

RECAI Revista de Estudios en Contaduría, Administración e Informática, vol. 8, núm. 23, 2019

Universidad Autónoma del Estado de México, México

Copyright 2019 Universidad Autónoma del Estado de México

Esta obra está bajo una Licencia Creative Commons Atribución-NoComercial-SinDerivar 4.0 Internacional. 


\section{Alienación y satisfacción laboral en la industria de vestiduras automotrices: Caso Ciudad Juárez, Chihuahua}

Work alienation and job satisfaction in the automotive garment industry: The case of Ciudad Juárez, Chihuahua.

Oscar Galván Mendoza

Universidad Autónoma de Baja California. , México

Recepción: 12 Junio 2019

noccarempo@gmail.com

Aprobación: 28 Agosto 2019

Armando Esquinca Moreno

Universidad Autónoma de Chihuahua., México

Recepción: 12 Junio 2019

Aprobación: 28 Agosto 2019

\section{RESUMEN:}

El objetivo de esta investigación fue analizar la relación entre la alienación laboral y la satisfacción laboral de trabajadores mexicanos en una empresa de manufactura de vestiduras automotrices en Ciudad Juárez, Chihuahua. La muestra del estudio incluyó 150 trabajadores mexicanos pertenecientes a los mandos medios y superiores de dicha organización. La investigación se caracterizó por tener un enfoque cuantitativo, un diseño no experimental, relacional y exploratorio con una dimensión temporal transversal. El análisis de los datos se llevó a cabo mediante el modelado de ecuaciones estructurales utilizando Mínimos Cuadrados Parciales (PLS). Los resultados obtenidos indicaron que la alienación laboral se relaciona negativa y significativamente con la satisfacción laboral.

Palabras Clave: Alienación laboral, satisfacción laboral, PLS.

\section{Abstract:}

The purpose of this research was to analyze the relationship between work alienation and job satisfaction in a sample of Mexican workers from an automotive garment company in Ciudad Juárez, Chihuahua. The sample of the study included 150 employees from the middle and upper management echelons in the organization under analysis. The research is characterized by having a quantitative approach, a non-experimental, relational and exploratory design with a cross-sectional temporal dimension. The data analysis was carried out by modeling structural equations using Partial Least Squares (PLS). The results obtained indicated that work alienation is negatively and significantly related to job satisfaction.

KEYWORDS: Work alienation, job satisfaction, PLS, Work alienation, job satisfaction, PLS.

\section{INTRODUCCIÓN}

En los últimos años, se han ido presentado numerosos e importantes cambios dentro del entorno y la estructura de la fuerza del trabajo (Martín, Salanova y Peiró, 2003), en donde la globalización de la economía, la flexibilización del trabajo, la rápida expansión del sector servicios y el incremento de la utilización de tecnología de la información influyen en la conducta de empleados, grupos de trabajo y organizaciones (Peiró, 2001). Ante esta situación, se debe hacer mención de una problemática latente que daña el rendimiento en las organizaciones: la alienación laboral (Mottaz, 1981; Sarros, Tanewski, Winter, Santora y Densten, 2002).

La alienación laboral se define como un sentimiento de decepción que provoca una desconexión psicológica por parte de un empleado hacia la organización a la que pertenece y las recompensas que ésta le ofrece (Kanungo, 1979; Moch, 1980). Adicionalmente, este fenómeno se relaciona directamente con las

\section{Notas DE AUtor}


condiciones laborales en las que se encuentra un empleado, dado que puede aislar al mismo en su entorno y de las actividades que debe realizar (Nair y Vohra, 2009). Es importante brindarle la debida atención, ya que es una variable que puede influir considerablemente en la productividad del empleado, considerada como crucial para el éxito de una organización (Galván, 2019), afectando negativamente a los indicadores de nivel de trabajo, principalmente a la satisfacción laboral (Blauner, 1964; Kanungo, 1982).

Por otro lado, no debe ignorarse que en la actualidad, la importancia del estudiar a la satisfacción laboral radica en que ésta se desarrolla en la persona, de acuerdo a sus experiencias en el entorno laboral (Hegney, Plank y Parker 2006), teniendo la capacidad de impactar directamente al ambiente de trabajo y a la productividad de los integrantes de la organización (Chiang, Salazar y Núñez, 2007).

Es por lo antes descrito lo que permitió que la presente investigación tuviera como objetivo el analizar la relación entre la alienación laboral y la satisfacción laboral de trabajadores mexicanos de una empresa de manufactura de vestiduras automotrices en Ciudad Juárez, Chihuahua.

\section{Eje TeóRico}

\subsection{Alienación laboral}

La alienación laboral es definida por Kanungo (1979) como el estado general de desconexión psicológica del trabajo generado por una decepción, el cual condiciona el potencial del trabajador para satisfacer sus propias necesidades y expectativas más destacadas. El principal precursor en los estudios de alienación laboral es Seeman (1959), quien estableció que la alienación es un constructo multidimensional, el cual se compone de cinco dimensiones: impotencia (sentimiento que presenta un trabajador sobre la ausencia de control sobre el desempeño de su trabajo diario), insignificancia (el trabajador piensa que su labor genera una escasa influencia sobre el proceso de producción de la organización), ausencia de normas (el trabajador siente que las normas son escasas y que han perdido su valor en su entorno laboral), aislamiento (ausencia de identificación del trabajador con su organización) y ausencia de autorrealización (el trabajador no se sienten realizado con la labor que desempeñan en la misma).

Bajo esa óptica, autores como Nair y Vohra (2009) y Chiaburu, Thundiyil y Wang (2014) caracterizan al trabajo de Seeman (1959) como ambiguo, ya que no incluye a los aspectos estructurales de la organización, la naturaleza del trabajo, las relaciones laborales y las percepciones con respecto a la justicia como parte de un constructo teórico central para el abordaje de la alienación que se presenta dentro de un contexto organizacional.

Con referencia a lo ya expuesto y a efecto de entender la relación propuesta entre las variables de alienación laboral y satisfacción del trabajador, se utiliza a la teoría del intercambio social como sustento del abordaje de la investigación. Dicha teoría explica que la vida social es un constante fluir de intercambios, en donde los individuos se saben necesitados de los demás para perpetuar los beneficios que reciben (Gouldner, 1960; Zoghbi y Caamaño, 2010). Con base en la evidencia científica empírica, se puede afirmar que los empleados pueden mostrar un comportamiento positivo o negativo como respuesta al tratamiento recibido de sus organizaciones (Greenberg y Scott, 1996). Las relaciones de intercambio que se establecen entre el trabajador y los superiores deben ser caracterizadas por brindar un trato justo y con calidad (Bies y Moag, 1986); se destaca la importancia del impacto que puede llegar a generar tanto en los resultados como en múltiples acciones y prácticas organizacionales (Blau, 1964).

Por otra parte, cabe señalar que, las consecuencias que han sido relacionadas con la alienación laboral a nivel personal son: generación de sentimientos de incapacidad, futilidad y aislamiento en el trabajador, aumento en la distracción del mismo, incidencia en conductas destructivas, aparición de estrés entre los trabajadores (Zoghbi y Caamaño, 2010). Hay que mencionar también que la alienación laboral se vincula directamente con el entorno organizacional debido a que influye en la disminución del compromiso y de la satisfacción laboral en los integrantes de una organización (Chiaburu et al., 2014). Y es que dado que este fenómeno 
alude a los sentimientos de un trabajador sobre una ausencia de control sobre el desempeño de su trabajo diario, también puede alterar negativamente la forma de interactuar de un individuo con sus compañeros de trabajo (Kanungo, 1982). A un largo plazo conlleva ausentismo, intención de renunciar, tensión y hasta el padecimiento del síndrome de quemarse por el trabajo (Chiaburu et al., 2014).

\subsection{Satisfacción laboral}

Partiendo de la idea de que el tema de la satisfacción laboral es de gran interés porque indica la habilidad de la organización para satisfacer las necesidades de los trabajadores (Galván, 2019) y porque existe evidencia que demuestra que los trabajadores insatisfechos faltan a trabajar con más frecuencia y suelen renunciar más, mientras que los empleados satisfechos gozan de mejor salud y viven más años (Alonso, 2008); la satisfacción laboral es entendida como aquellas orientaciones afectivas por parte de los individuos hacia los roles de trabajo que ocupan (Vroom, 1964). Por su parte, Hoppock (1935) le define como cualquier combinación de factores psicológicos, fisiológicos y circunstancias ambientales que hacen que una persona se sienta satisfecha con su trabajo. Teniendo en consideración ambas perspectivas, no se debe olvidar que la satisfacción laboral es una variable importante para las organizaciones porque tiene impactos significativos en el desempeño laboral, (Singh y Loncar, 2010), en la productividad y en el bienestar personal (Islam, Mohammad y Ahmed, 2013).

\subsection{Relación entre alienación laboral y satisfacción del trabajador}

Los estudios sobre alienación laboral han encontrado que dicha variable afecta negativamente a los indicadores de nivel de trabajo, principalmente a la satisfacción laboral (Blauner, 1964; Kanungo, 1982). Bajo ese razonamiento, es necesario comprender que los trabajadores que están alienados normalmente experimentan una menor satisfacción laboral porque están alejados de sus trabajos, menos vinculados a ellos y a la organización en la que se desempeñan (Marshall, Michaels y Mulki, 2007; Armstrong, 2004). De ahí que se afirme que la alienación laboral tiene consecuencias negativas sobre las actitudes de los trabajadores, emergiendo como un importante predictor de la satisfacción laboral (Chiaburu et al., 2014).

Similarmente, Schultz y Schultz (1990) detallan que la alienación laboral puede tener implicaciones de índole emocional y puede causar la disminución de la satisfacción en cada paso de la vida de un trabajador. Considerando que dentro de la investigación sustancial sobre los efectos de la alienación laboral se detecta una influencia negativa hacia la satisfacción laboral (Bacharach y Aiken, 1979; McKinlay y Marceau, 2011), no se debe olvidar que aunque la alienación es un estado personal, los empleados alienados perjudican al funcionamiento de la organización (Brender-Ilan, 2012).

De lo anterior se deriva la necesidad de presentar de manera detallada a aquellos estudios que se han publicado en los últimos cinco años y que han analizado la relación entre la alienación laboral y la satisfacción laboral para justificar la vigencia del tema y sustentar su abordaje respectivo:

1) La investigación de los autores Siron, Muttar, Ahmad y Tarsipan (2016), la cual se concentra especialmente en el estudio de trabajadores académicos y profesores de colegios e institutos técnicos en Iraq, encontrando que la alienación laboral tiene un impacto negativo en la satisfacción laboral de los mismos.

2) El estudio de Fedi, Pucci, Tartaglia y Rollero (2016) afirma que la alienación que se presenta dentro de una organización disminuye tanto la satisfacción laboral como la participación en el trabajo de los empleados que integran a la misma, fue desarrollado en Italia en trabajadores de altos mandos de empresas dedicadas a brindar servicios, como hospitales, hoteles y bancos.

3) El documento de Kurdi (2018) resalta el impacto negativo de la alienación laboral en el desempeño y la satisfacción laboral de médicos de un complejo medico de Palestina.

4) La investigación de Özer, Uğurluoğlu, Saygılı, y Sonğur (2019) explica la disminución de la satisfacción laboral de enfermeras y psiquiatras de un hospital público en Turquía como consecuencia del impacto por parte de la alienación laboral.

5) La tesis doctoral de Galván (2019), en donde se muestra que la alienación laboral se asocia estadística y significativamente con la satisfacción laboral, con base en la perspectiva de trabajadores de mandos medios y 
superiores que pertenecen a empresas de manufactura de arneses automotrices en Ciudad Juárez, Chihuahua, México.

Otro estudio identificado y que fue llevado a cabo en México, específicamente en el Estado de México, fue el de García y Fierro (2019). Dicha investigación no aborda relación planteada líneas arriba ni considera a la población que pertenece a la industria manufacturera pero, sirve como antecedente y sustento para una mejor comprensión del fenómeno de la alienación laboral desde un enfoque organizacional en el sector público en México.

\subsection{Industria de vestiduras automotrices en Ciudad Juárez}

Ciudad Juárez, situada en la frontera norte de México, es un polo de desarrollo económico del país; su concentración industrial es de 332 empresas instaladas, las cuales se dedican a la manufactura de diferentes productos, teniendo una ocupación de 302,654 personas (INDEX, 2019a). Con base en Salgado (29 de mayo de 2019), las industrias maquiladoras en Ciudad Juárez han mostrado el interés de dar seguimiento y atender problemáticas que atañen a la salud mental de los trabajadores, considerado como un aspecto esencial. La misma autora señala que, al no atender este rubro, las consecuencias a largo plazo pueden ser: reducción de productividad, descenso de calidad de vida, problemas de salud física o mental (enfermedades), trastornos de depresión y ansiedad, problemas familiares y riesgos de adicciones.

Considerando que las empresas manufactureras que pertenecen al sector automotriz tienen un gran impacto en la economía de Ciudad Juárez al representar el 32\% del total de empresas registradas (INDEX, 2019b), es necesario precisar que las empresas de costura de vestidura automotriz se caracterizan por registrar una de las tasas más altas de rotación de personal, el registro más reciente presenta un $6.44 \%$ al término del mes de junio de 2019; también, fue en ese mismo en donde se identificó una tasa de ausentismo de .94\% (INDEX, 2019c).

Acorde al análisis de la evidencia científica disponible que versa sobre la alienación laboral, se detectó la ausencia de estudios similares con muestras mexicanas que pudieran caracterizar y evidenciar la relación entre las variables ya mencionadas.

Ante esta situación, cabe preguntarse ¿Cómo es la relación entre la alienación laboral y la satisfacción laboral de trabajadores mexicanos de una empresa de manufactura de vestiduras automotrices en Ciudad Juárez, Chihuahua?. De ahí que este estudio buscó analizar la relación entre la alienación laboral y la satisfacción laboral específicamente en una muestra de trabajadores mexicanos de una empresa dedicada a la manufactura de vestiduras automotrices en Ciudad Juárez, Chihuahua.

Dicho lo anterior, se formuló la siguiente hipótesis:

H1. La alienación laboral se relaciona negativa y significativamente con la satisfacción laboral

\section{Método de inVestigación}

Con el fin de alcanzar el objetivo, la investigación se caracterizó por tener un enfoque cuantitativo, un diseño no experimental, relacional y exploratorio con una dimensión temporal transversal. Los datos utilizados en la investigación fueron obtenidos a través de un cuestionario que se les aplicó a los trabajadores pertenecientes a los mandos medios y superiores que laboran en empresas dedicadas a la manufactura de vestiduras automotrices en Ciudad Juárez, Chihuahua. Se contemplaron a los mandos medios y superiores (técnicos, coordinadores, supervisores y gerentes) debido a que se les considera los gestores de la organización y quienes la orientan hacia los objetivos definidos por la empresa (Galván, 2019). En relación con la muestra, ésta fue por conglomerados, en función de que la unidad muestral fue un grupo de elementos que contuvo representantes de toda la población necesaria para cumplir con el objetivo del estudio (Izquierdo, 2012). Acorde con Triola (2009), el muestreo por conglomerados se caracteriza por ser homogéneo entre sí, pero internamente presenta un alto grado de heterogeneidad en sus componentes. 
Los datos fueron recolectados en el mes de noviembre de 2018; en total se recolectaron 166 cuestionarios, de los cuales se descartaron 16 por no ser llenados en su totalidad, lo que dejó un total de 150 cuestionarios utilizables para el análisis de esta investigación.

Las características demográficas de los participantes se presentan en la Tabla 01. Con base en los resultados obtenidos, dentro del apartado del sexo de los participantes, de las 150 que participaron, el $45.3 \%$ son mujeres y el $54.7 \%$ son hombres. El segundo apartado es el de la edad, en donde los participantes menores de 20 años conforman un $12.7 \%$ del total de la muestra, los que se encuentran entre los rangos de 20 a 50 años representan un $68.1 \%$ y aquellos participantes pertenecientes al rango de mayores de 50 años se identifica un 19.2\% del total de los participantes. Con respecto al apartado de la antigüedad laboral, se puede observar que un $9.3 \%$ de los participantes tiene menos de 1 año trabajando para la organización, un $55.4 \%$ refiere tener entre 1 y 4 años de antigüedad y un 35.3\% reporta más de 5 años de trabajo en la organización respectiva. En lo referente al apartado de los puestos, destacan el de técnico con un $48 \%$, el de supervisor con un $39.3 \%$ y el de gerente con un $12.7 \%$.

TABLA 01

Características demográficas de los participantes $(\mathrm{n}=150)$

Fuente: Elaboración propia.

3.1 Medición de los constructos

A partir del análisis de la evidencia científica fue posible identificar los instrumentos de medición que fueron utilizados para evaluar las variables que conforman esta investigación. Posteriormente, se procedió a la operacionalización de los mismos para lo cual se adaptó y construyó un instrumento de medición el cual fue administrado directamente a los empleados en su contexto laboral.

\subsubsection{Constructo: Alienación laboral.}

Se eligió la escala New Measure of Work Alienation de Nair y Vohra (2009), el cual es un instrumento auto-administrado que consta de 08 preguntas. Una vez seleccionada la escala, se solicitó la autorización de los autores para usarla y adaptarla al contexto mexicano. Para responder a los ítems del constructo, el valor mínimo de respuesta fue 1 [Totalmente en desacuerdo] y el valor máximo fue 6 [Totalmente de acuerdo].

3.1.2 Constructo: Satisfacción laboral.

Para medir a la satisfacción laboral se utilizó el Cuestionario de Satisfacción Laboral S20/23 de Meliá y Peiró (1989), el cual es constituido por 23 ítems que abordan la información objetiva del trabajo y aquellos elementos subjetivos asociados a diferentes aspectos del trabajo (Jiménez, Jara y Miranda, 2012). De igual forma se solicitó la autorización de los autores para usar el cuestionario dentro del contexto mexicano. Por otra parte, en lo que corresponde a las opciones de respuesta a los ítems del constructo, el valor mínimo de respuesta fue 1 [Muy insatisfecho] y el valor máximo fue 6 [Muy satisfecho].

3.2 Técnica de análisis de datos

Los datos se analizaron mediante la técnica estadística de modelado estructural basado en mínimos cuadrados parciales. La modelación de ecuaciones estructurales basada en mínimos cuadrados parciales, nombrada [PLS] por sus siglas en inglés -Partial least squares- (Wold, Esbensen y Geladi, 1987), es una técnica que emergió "para analizar las complejas relaciones entre variables latentes que permiten explicar los datos observados y el análisis predictivo como elemento relevante en la investigación científica” (Martínez y Fierro, 2018:134).

Se considero adecuado utilizar el modelado PLS dado que la investigación incluye a una variable que se encuentra en una etapa temprana de desarrollo teórico (la alienación laboral). Además, Henseler, Ringle y Sinkovics (2009) le consideran como óptimo para probar y validar modelos exploratorios, ya que este tipo de modelado está orientado a la predicción y puede manejar modelos de medición tanto reflectivos como formativos. Bajo esa misma línea de razonamiento, Chin, Peterson y Brown (2008) hacen mención que una 
de las principales características del PLS es que permite estimar un modelo con un gran número de variables e indicadores latentes con un tamaño de muestra pequeño, precisamente el caso de este estudio, al tener como muestra a 150 participantes.

Aunado a lo anterior, cabe resaltar que el modelado PLS se basa en análisis de la varianza y tiene suposiciones menos estrictas sobre la distribución de variables y términos de error (Henseler et al., 2009), estimando parámetros similares a los componentes principales con un enfoque de regresión múltiple (Hsu, Chen, y Hsieh, 2006), por lo que permite confirmar teoría, además de sugerir relaciones entre variables que podrían existir o no y plantear dado el caso, propuestas para futuros vínculos entre constructos (Chin, 1998; Reinartz, Haenlein y Henseler, 2009).

Para el modelado de ecuaciones estructurales basado en mínimos cuadrados parciales se utilizó el software SmartPLS 3 de Ringle, Wende y Becker (2015).

\section{ANÁlisis E INTERPRETACión DE RESULTADOS}

\subsection{Fiabilidad y validez de los indicadores}

La fiabilidad de los indicadores especifica qué parte de la varianza de un indicador se puede explicar por la variable latente (Gotz, Liehr-Gobbers y Krafft, 2010), por lo que el criterio utilizado para evaluar la fiabilidad del indicador es la carga factorial (Seidel y Back, 2009). Al evaluar las cargas factoriales, el investigador debe saber que se aceptarán aquellos ítems con cargas iguales o superiores a .0.70 (Henseler et al., 2009). No obstante, cuando existan otros indicadores en el mismo constructo que presenten cargas altas, un ítem con valor de .50 puede ser válido y aceptarse (Chin, 1998; Hair, Sarstedt, Pieper y Ringle, 2012); dentro de esta investigación se consideró el criterio anterior.

Para la evaluación del modelo se realizó una corrida datos, donde se analizó las cargas factoriales de todos los ítems, detectando que ninguno de los mismos presento cargas inferiores a .50. A continuación se presenta la Tabla 02, la cual contiene las cargas factoriales correspondientes al modelo de medición, el valor $\mathrm{t}$ y su respectiva significancia. En lo que atañe a la significancia estadística de las cargas, ésta es de $99.9 \%$ en todos los casos cuando el valor $t$ es mayor que 3.291. 
Tabla 02: Cargas y valores t de los indicadores $(n=150)$

\begin{tabular}{|c|c|c|c|}
\hline Constructo & Ítem & Cargas & Valor $\mathrm{t}$ \\
\hline & Alien 1 & 0.776 & $14.278^{*}$ \\
\hline & Alien2 & 0.836 & $21.726^{*}$ \\
\hline & Alien3 & 0.864 & $35.893^{*}$ \\
\hline Alienación & Alien 4 & 0.769 & $18.287^{*}$ \\
\hline \multirow[t]{14}{*}{ laboral } & Alien5 & 0.688 & $15.02^{*}$ \\
\hline & Alien6 & 0.789 & $18.855^{*}$ \\
\hline & Alien7 & 0.880 & $36.298^{*}$ \\
\hline & Alien8 & 0.772 & $18.496^{*}$ \\
\hline & Sat1 & 0.603 & $9.389^{*}$ \\
\hline & Sat10 & 0.557 & $9.284^{*}$ \\
\hline & Sat11 & 0.666 & $11.754^{*}$ \\
\hline & Sat12 & 0.624 & $9.768^{*}$ \\
\hline & Sat13 & 0.706 & $13.379^{*}$ \\
\hline & Sat14 & 0.767 & $19.045^{*}$ \\
\hline & Sat15 & 0.674 & $10.589^{*}$ \\
\hline & Sat16 & 0.700 & $13.684^{*}$ \\
\hline & Sat17 & 0.772 & $19.541^{*}$ \\
\hline & Sat18 & 0.824 & $24.655^{*}$ \\
\hline \multirow{13}{*}{$\begin{array}{l}\text { Satisfacción } \\
\text { laboral }\end{array}$} & Sat19 & 0.763 & $18.889^{*}$ \\
\hline & Sat2 & 0.664 & $13.079^{*}$ \\
\hline & Sat20 & 0.767 & $17.774^{*}$ \\
\hline & Sat21 & 0.719 & $14.256^{*}$ \\
\hline & Sat22 & 0.722 & $17.06^{*}$ \\
\hline & Sat23 & 0.764 & $18.06^{*}$ \\
\hline & Sat3 & 0.643 & $12.74^{*}$ \\
\hline & Sat4 & 0.553 & $10.199^{*}$ \\
\hline & Sat5 & 0.705 & $18.834^{*}$ \\
\hline & Sat6 & 0.605 & $9.522^{*}$ \\
\hline & Sat7 & 0.592 & $9.286^{*}$ \\
\hline & Sat8 & 0.614 & $9.775^{*}$ \\
\hline & Sat9 & 0.557 & $8.787^{*}$ \\
\hline \multicolumn{4}{|c|}{$\begin{array}{l}\text { "Significativo con un valor } p \text { de } 0.000 \text {, si } t>3.291 \text {, para una } \\
\text { prueba de dos colas }\end{array}$} \\
\hline
\end{tabular}

Acorde al contenido de la Tabla anterior, los valores t de los ítems que conforman los diferentes constructos se encuentran en un rango de 8.787 [Sat9] a 36.298 [Alien7], es decir, todas las cargas resultaron significativas estadísticamente, evidenciando valores t superiores a 3.291.

4.2 Confiabilidad

La confiabilidad o fiabilidad, se refiere a la consistencia o estabilidad de una escala de medición, se mide a través del alfa de Cronbach (Quero, 2010). Un alto valor alfa supone que los puntajes de todos los elementos dentro de un constructo tienen el mismo rango y significado (Cronbach, 1951). Debe ser mayor a 0.7, aunque en estudios exploratorios 0.6 es aceptable (Hair, Black, Babin y Anderson, 2010). Sin embargo, como el alfa de cronbach tiende a proporcionar una subestimación severa de la confiabilidad de la consistencia interna de las variables latentes en los modelos path PLS, es más apropiado aplicar una medida diferente, la confiabilidad compuesta (Werst, Linn y Jöreskog, 1974). La fiabilidad compuesta tiene en cuenta que los indicadores tienen cargas diferentes, y se pueden interpretar de la misma manera que alfa de Cronbach (Henseler et al., 2009), no importa qué coeficiente de confiabilidad particular se use, un valor de fiabilidad de consistencia interna superior a 0.7 en etapas tempranas de investigación y valores superiores a 0.8 o 0.9 en etapas más avanzadas de investigación se consideran satisfactorios (Nunnally y Bernstein, 1994), mientras que un valor inferior a 0.6 indica una falta de fiabilidad. En la Tabla 03 se presentan los estadísticos de fiabilidad compuesta y el alfa de Cronbach. En todos los casos las variables latentes exceden el punto de corte de 0.70 
Tabla 03: Estadísticos de fiabilidad del modelo

\begin{tabular}{lll}
\hline Constructo & $\begin{array}{l}\text { Alfa de } \\
\text { Cronbach }\end{array}$ & Fiabilidad compuesta \\
\hline Alienación Laboral & .918 & .933 \\
Satisfacción laboral & .946 & .952 \\
\hline
\end{tabular}

Fuente: Elaboración propia a partir de resultados de SmartPLS 3.

\subsection{Validez de convergencia}

La validez de convergencia muestra el grado de representación que tienen los indicadores con respecto a su constructo, por lo que calcula el porcentaje de varianza interpretado por los factores latentes del error de medición (Shyu, Li y Tang, 2013). Acorde a Campbell y Fiske (1959), para que exista validez convergente los indicadores de un mismo constructo deben correlacionar altamente entre ellos. Por ello, es necesario recalcar que la validez de convergencia se calcula mediante el promedio de la varianza extraída [Average Variance Extracted, AVE por sus siglas en ingles] (Fornell y Larcker, 1981). En términos generales, el criterio para la varianza promedio extraída debe ser mayor que 0.5 ; en caso de obtener más de 0.5 en el promedio de varianza extraída, se puede apreciar que la construcción es más confiable (Hair, Black, Babin, Anderson y Tatham, 2005; Wu, 2008). La Tabla 04 muestra el análisis de varianza extraída de los constructos respectivos:

Tabla 04: Análisis de varianza extraída de los constructos.

\begin{tabular}{ll}
\hline Constructo & AVE \\
\hline Alienación Laboral & .638 \\
Satisfacción laboral & .564 \\
\hline
\end{tabular}

Fuente: Elaboración propia a partir de resultados de SmartPLS 3.

4.4 Validez discriminante

La validez discriminante asegura que un constructo sea empíricamente único y represente un fenómeno de interés que otras medidas en un modelo de ecuación estructural no puedan capturar (Hair et al., 2010). Técnicamente, la validez discriminante muestra cuando cada elemento de medición de un constructo se correlaciona débilmente con el resto de los constructos, excepto con aquellos que se asocia teóricamente (Gefen y Straub, 2005). Si no se establece la validez discriminante, "los constructos tienen una influencia en la variación de algo más que las variables observadas con las que están relacionadas teóricamente" (Farrell 2010: 324). Para poder evaluar la validez discriminante se debe analizar la raíz cuadrada del promedio de la varianza extraída de cada constructo; ésta debe ser mayor a los niveles de correlación del constructo (Fornell y Larcker, 1981).

Se presenta a continuación la matriz de correlación, en cuya diagonal se observa el promedio de la varianza extraída para cada una de las variables latentes de esta investigación [ver Tabla 5]. De acuerdo al análisis los datos, se detecta que los valores AVE son superiores a las correlaciones entre constructos, por lo que se afirma que existe validez discriminante.

Tabla 5: Matriz de correlaciones entre constructos y AVE (Criterio Fornell - Larcker).

\begin{tabular}{lll}
\hline Constructo & Alienación Laboral & $\begin{array}{l}\text { Satisfacción } \\
\text { laboral }\end{array}$ \\
\hline Alienación Laboral & .799 & \\
Satisfacción laboral & -.685 & .750 \\
\hline
\end{tabular}


Fuente: Elaboración propia a partir de resultados de SmartPLS 3.

Considerando que la validez discriminante indica en qué medida un constructo determinado es diferente de otros constructos, son Martínez y Fierro (2018) quienes afirman que, para poder valorar la validez discriminante es necesario evaluar tres criterios: 1) criterio de Fornell-Larcker, 2) cargas cruzadas entre indicadores y variables latentes y 3 ) la matriz de correlaciones HTMT.

Con respecto a las cargas cruzadas de los indicadores del modelo de esta investigación, dentro de la Tabla 06 se puede apreciar que en todos los casos las cargas de los ítems con sus respectivos constructos son mayores a 0.50 , por lo que se afirma que existe validez convergente. Sumado a ello, se detecta que las cargas de los ítems con otros indicadores son débiles, por lo cual se afirma que existe validez discriminante.

Tabla 06: Cargas cruzadas de los indicadores y variables latentes del modelo.

\begin{tabular}{lll}
\hline İtem & Alienación laboral & Satisfacción laboral \\
\hline Alien1 & 0.776 & -0.505 \\
Alien2 & 0.836 & -0.557 \\
Alien3 & 0.864 & -0.598 \\
Alien4 & 0.769 & -0.584 \\
Alien5 & 0.688 & -0.393 \\
Alien6 & 0.789 & -0.497 \\
Alien7 & 0.880 & -0.598 \\
Alien8 & 0.772 & -0.599 \\
Sat1 & -0.532 & 0.603 \\
Sat10 & -0.351 & 0.557 \\
Sat11 & -0.439 & 0.666 \\
Sat12 & -0.324 & 0.624 \\
Sat13 & -0.464 & 0.706 \\
Sat14 & -0.511 & 0.767 \\
Sat15 & -0.474 & 0.674 \\
Sat16 & -0.432 & 0.700 \\
Sat17 & -0.491 & 0.772 \\
Sat18 & -0.471 & 0.824 \\
Sat19 & -0.48 & 0.763 \\
Sat2 & -0.536 & 0.664 \\
Sat20 & -0.46 & 0.767 \\
Sat21 & -0.377 & 0.719 \\
Sat22 & -0.424 & 0.722 \\
Sat23 & -0.485 & 0.764 \\
Sat3 & -0.537 & 0.643 \\
Sat4 & -0.383 & 0.553 \\
Sat5 & -0.569 & 0.705 \\
Sat6 & -0.49 & 0.605 \\
Sat7 & -0.464 & 0.592 \\
Sat8 & -0.467 & 0.614 \\
Sat9 & -0.372 & 0.557 \\
\hline & &
\end{tabular}

Fuente: Elaboración propia a partir de resultados de SmartPLS 3.

En lo que atañe a las correlaciones Heterotrait-Monotrait (HTMT), Henseler, Ringle y Sarstedt (2015) les describen como un nuevo método para evaluar la validez discriminante en PLS-SEM, siendo uno de los componentes clave de la evaluación del modelo. Y es que si no se establece la validez discriminante, los investigadores no pueden confirmar que los resultados del modelo estructural son reales, o si son simplemente el resultado de inconsistencias estadísticas (Imran, Aziz y Hamid, 2017). De ahí que Hair Jr, Hult, Ringle y Sarstedt (2016) afirmen que el criterio HTMT supera claramente a los enfoques estándar para la evaluación de la validez discriminante, como el criterio de Fornell y Larcker y las cargas cruzadas, que son ciertamente incapaces de detectar una falta de validez discriminante.

Con respecto al valor de la relación HTMT, éste debe estar por debajo de 1 (Imran, Aziz y Hamid, 2017). Por otro lado, autores como Gold, Malhotra y Segars (2001) consideran un valor de 0.90 como el más 
apropiado, por lo que en el estudio actual se encontró que todos los valores de HTMT son menores que .90, validando todas las construcciones de estudio relacionadas con la validez discriminante [ver Tabla 07].

Tabla 07: Matriz de ratios Heterotrait-Monotrait (HTMT).

\begin{tabular}{lll}
\hline Constructo & Alienación Laboral & $\begin{array}{l}\text { Satisfacción } \\
\text { laboral }\end{array}$ \\
\hline Alienación Laboral & & \\
Satisfacción laboral & .718 & \\
\hline
\end{tabular}

Fuente: Elaboración propia a partir de resultados de SmartPLS 3.

4.5 Análisis de multicolinealidad

Para poder identificar la existencia de multicolinealidad entre los constructos, se contempló la utilización del estadístico FIV, el factor de inflación de la varianza (VIF, por sus siglas en inglés). La función de dicho factor es la de revisar los problemas de multicolinealidad, es decir, identifica la presencia de coeficientes de regresión inflados cuando las variables están altamente correlacionadas entre sí (Quevedo, García, Salas, Domínguez y Esquivel, 2007).

Particularmente, cuando los indicadores son reflectivos, Montgomery y Runger (2008) señalan que la presencia de multicolinealidad se puede observar fácilmente por la magnitud del factor de inflación de la varianza de cada ítem, especificando que si el valor fiv del ítem es superior 10, la multicolinealidad será un problema. De acuerdo con los datos que contiene la Tabla 08, se observa que ninguno de los indicadores reflectivos está por arriba de 10, por lo cual se puede afirmar que no existen problemas de multicolinealidad.

Tabla 08: Factores de inflación de la varianza (FIVs) de las variables del modelo

\begin{tabular}{|c|c|c|c|c|c|}
\hline Constructo & İtem & FIV & Constructo & Ítem & FIV \\
\hline \multirow{8}{*}{$\begin{array}{l}\text { Alienación } \\
\text { laboral }\end{array}$} & Alien 1 & 2.832 & \multirow{16}{*}{$\begin{array}{l}\text { Satisfacción } \\
\text { laboral }\end{array}$} & Sat16 & 2.691 \\
\hline & Alien2 & 3.28 & & Sat17 & 3.223 \\
\hline & Alien3 & 3.195 & & Sat18 & 4.573 \\
\hline & Alien 4 & 2.197 & & Sat19 & 3.096 \\
\hline & Alien5 & 1.886 & & Sat2 & 4.06 \\
\hline & Alien6 & 3.165 & & Sat20 & 4.019 \\
\hline & Alien7 & 4.931 & & Sat21 & 3.315 \\
\hline & Alien8 & 2.305 & & Sat22 & 2.933 \\
\hline \multirow{8}{*}{$\begin{array}{l}\text { Satisfacción } \\
\text { laboral }\end{array}$} & Sat1 & 2.532 & & Sat23 & 3.196 \\
\hline & Sat10 & 2.74 & & Sat3 & 2.991 \\
\hline & Sat11 & 3.151 & & Sat4 & 1.877 \\
\hline & Sat12 & 2.56 & & Sat5 & 2.316 \\
\hline & Sat13 & 3.648 & & Sat6 & 2.267 \\
\hline & Sat14 & 5.266 & & Sat7 & 3.03 \\
\hline & Sat15 & 3.628 & & Sat8 & 3.448 \\
\hline & & & & Sat9 & 3.481 \\
\hline
\end{tabular}

Fuente: Elaboración propia a partir de resultados de SmartPLs 3.

Fuente: Elaboración propia a partir de resultados de SmartPLS 3.

Dentro del modelado de ecuaciones estructurales se utiliza a la rho_A como una medida de fiabilidad consistente de las puntuaciones de los constructos (Dijkstra y Henseler, 2015). Dicha medida proporciona una estimación más precisa de la consistencia de los datos y de los valores que presenta cada constructo, indicando si son confiables, basándose en las cargas en lugar de las correlaciones identificadas entre las variables observadas (Henseler, 2017). 
Chin (1998) recomienda que los puntajes aceptables para el rho_A deben ser superiores a 0.70. En esta investigación, se obtuvieron resultados muy por encima del punto de corte de .70 en cada uno de los constructos [ver Tabla 09].

Tabla 09: Indicador de confiabilidad rho_A

\begin{tabular}{lc}
\multicolumn{2}{l}{ Tabla 09: Indicador de confiabilidad rho_A } \\
\hline Constructo & rho_A \\
\hline Alienación Laboral & .924 \\
Satisfacción laboral & .948 \\
\hline Fuente: Elaboración propia a partir de resultados de \\
SmartPLS 3.
\end{tabular}

Fuente: Elaboración propia a partir de resultados de SmartPLS 3.

4.6 Evaluación del modelo estructural

Inicialmente se evaluó la significancia de los coeficientes path del modelo a través del proceso bootstrapping. Según Martínez y Fierro (2018), el bootstrapping es una técnica no paramétrica que se encargar de probar si los caminos entre variables son factibles, evaluando la precisión de las estimaciones de PLS. Al generar submuestras tomadas de la muestra original para proporcionar el error estándar, el bootstrapping devuelve una aproximación de valores t para evaluar la significancia del path estructural, donde el resultado se aproxima a la normalidad de los datos (Kwong-Kay, 2013). Es a partir del bootstrapping lo que permite generar coeficientes de regresión estandarizados, también conocidos como coeficientes path, éstos muestran el efecto directo de las variables independientes (exógena) sobre una variable dependiente (endógena) en el modelo estructural planteado (Levy, Varela y Abad, 2006).

Se corrió el algoritmo bootstrapping con 500 interacciones, métrica de datos de media 0 y varianza de 1 y se obtuvieron 500 submuestras, obteniendo el siguiente resultado [ver Tabla 10]:

Tabla 10: Evaluación de los efectos directos entre los constructos planteados

Tabla 10: Evaluación de los efectos directos entre los constructos planteados

\begin{tabular}{|c|c|c|c|c|c|}
\hline Hipótesis & $\begin{array}{l}\text { Coeficiente } \\
\text { path }\end{array}$ & $\begin{array}{l}\text { Estadístico } \\
\mathrm{T}\end{array}$ & $\mathrm{R} 2$ & $\begin{array}{l}\text { Valor } \\
\text { P }\end{array}$ & Resultado \\
\hline $\begin{array}{l}\text { H1. La alienación } \\
\text { laboral se } \\
\text { relaciona } \\
\text { negativa y } \\
\text { significativamente } \\
\text { con la } \\
\text { satisfacción } \\
\text { laboral. }\end{array}$ & -.685 & 12.55 & .470 & 0.000 & $\begin{array}{l}\text { No se } \\
\text { rechaza }\end{array}$ \\
\hline
\end{tabular}

Fuente: Elaboración propia a partir de resultados de SmartPLS 3.

Con base en el contenido de la Tabla anterior, es posible apreciar una fuerte relación entre la alienación laboral con la variable latente de satisfacción laboral, ya que se obtuvo un coeficiente path de -.685. Se debe recalcar que, dada la estandarización, los coeficientes path pueden variar de $-1 \mathrm{a}+1$, por lo que los pesos más cercanos al 1 absoluto, independientemente del signo algebraico, reflejan los caminos más fuertes (Garson, 2016). Adicionalmente, dicho coeficiente se caracteriza por ser estadísticamente significativo por su valor $\mathrm{t}$ $(t=12.55$ con un valor $\mathrm{p}$ de 0.000$)$, ya que es mayor al valor de 1.96 recomendado en la evidencia científica (Cohen, 1992). En lo que se refiere al signo algebraico del coeficiente path, éste coincide con el signo asignado 
a priori, lo que provee una validación empírica parcial de la relación teórica asumida entre las variables latentes (Henseler et al., 2009).

Posteriormente, es necesario mencionar que el coeficiente de determinación $\mathrm{R}^{2}$ indica la proporción de varianza explicada por el constructo exógeno en cada uno de los constructos endógenos (Levy, Varela y Abad, 2006). El modelo estructural de la presente investigación fue evaluado con los criterios de $\mathrm{R}^{2}$ en variables endógenas, considerando una $R^{2}$ de 0.67 como sustancial; una $R^{2}$ de 0.33 como moderada y una $R^{2}$ de 0.19 considerada débil (Henseler et al, 2009). La $\mathrm{R}^{2}$ obtenida como resultado fue de 0.470 (moderada), lo que significa que la variable de alienación laboral explica el $47 \%$ de la varianza del constructo de satisfacción laboral. Por lo que se concluye que la alienación laboral se caracteriza por ser una variable predictora al relacionarse negativa, estadística y significativamente con la satisfacción laboral, brindando el soporte necesario para no rechazar la hipótesis planteada en este estudio [Figura 1].

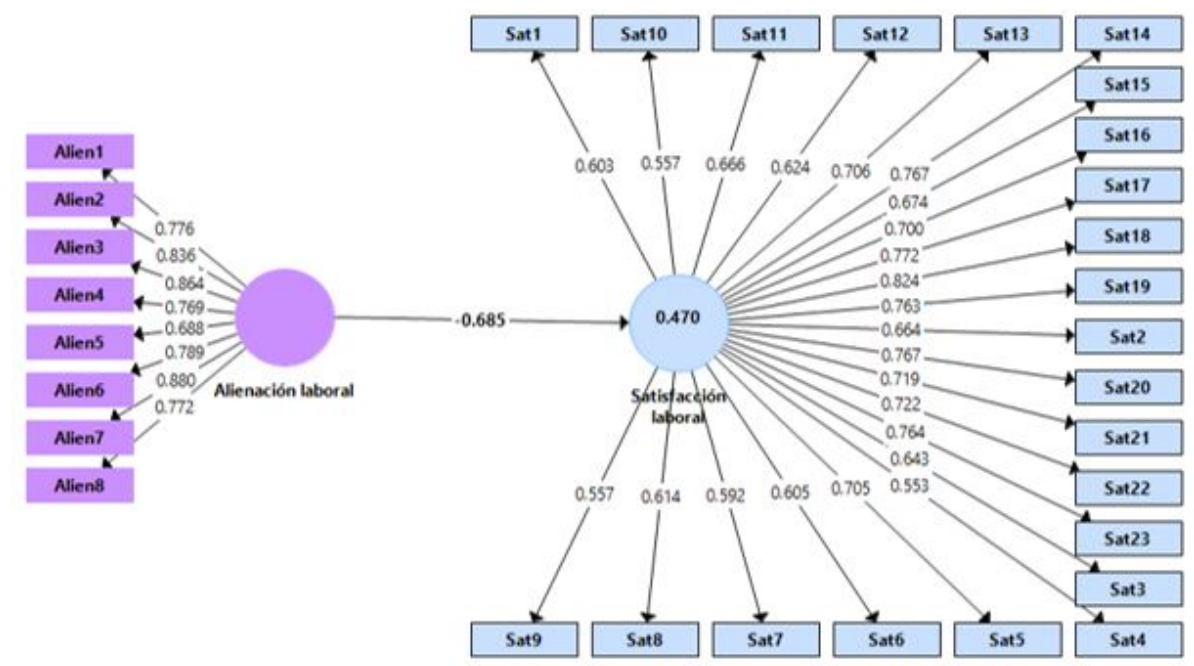

Figura 1: Modelo estructural

Fuente: Elaboración propia a partir de resultados de SmartPLS 3.

\section{Discusión}

Específicamente en una empresa de manufactura de vestiduras automotrices en Ciudad Juárez, Chihuahua, esta investigación buscó analizar la relación entre la alienación laboral y la satisfacción laboral de trabajadores mexicanos de mandos medios y superiores. Por tanto, al cuestionar respecto a cómo se relacionan ambas variables, los resultados indican la existencia de una relación negativa que se caracteriza por ser estadísticamente significativa.

La presente investigación proporciona evidencia que apoya la relación planteada entre las variables, de tal manera que es la alienación laboral se puede perfilar como una importante variable predictora de la satisfacción laboral dentro del contexto seleccionado para el estudio. Esto coincide con investigaciones como la de Sirin, Duman y Karakus (2011) quienes afirman que la magnitud de la relación entre ambas variables arroja como resultado una relación negativa y significativa. De ahí que cuando la alienación laboral se hace presente en los trabajadores, éstos no puedan satisfacer sus necesidades y expectativas sobresalientes del trabajo (Banai, Reisel y Probst, 2004). Sumado a ello, otra investigación identificada dentro del análisis de la evidencia científica y que sustenta la relación ya abordada es la de Chiaburu, Díaz y De Vos (2013), quienes explican que la alienación laboral también genera una influencia negativa en la satisfacción con el trabajo, afectando considerablemente en la carrera profesional de los empleados.

Hay que mencionar, además que el aporte empírico de Nasurdin, Ramayah y Kumaresan (2005) detalla que la alienación en el lugar de trabajo provoca que los empleados no sean capaces de satisfacer sus 
necesidades sociales. Similarmente, Ceylan y Sulu (2010) puntualizan que a través de una disminución de la motivación, la alienación laboral separa psicológicamente a las personas de su trabajo y actúa para reducir su satisfacción y participación laboral. Ciertamente, se establece que la alienación laboral puede repercutir en el funcionamiento de la empresa manufacturera dedicada a la costura de vestidura automotriz, generando distracción en el trabajador y sentimientos de incapacidad. Por otro lado, en esencia y coincidente con Seybolt y Gruenfeld (1976), la alienación laboral está directamente relacionada con las expectativas que un individuo tiene sobre su trabajo a partir de sus experiencias anteriores y la medida en que éste cumple esas expectativas. Por lo que cabe destacar que a un empleado alienado no le importa personalmente la participación en los procesos de trabajo, su objetivo primordial es únicamente el de ganar dinero (Shepard, 1970).

Con base en lo anterior, es necesario que las empresas dedicadas a la manufactura de vestiduras automotrices en Ciudad Juárez, Chihuahua conozcan y comprendan el impacto que puede tener una problemática latente como la alienación laboral, ya que es predominante la utilización de estrategias enfocadas en recompensar (monetariamente) el desempeño de los trabajadores sin contemplar sus necesidades de desarrollo psicológico y a la par, sin considerar y promover las aptitudes que tienen los mismos para desempeñar sus actividades. Bajo esa óptica, se recomienda desarrollar actividades relativas a la proporción de mayor autonomía y de retroalimentación, actividades que precisamente brindan un carácter dinámico haciendo que el entorno del trabajo sea más significativo e intrínsecamente satisfactorio.

No se debe olvidar que, es a través del otorgamiento de esas recompensas extrínsecas lo que permite que las organizaciones impidan que sus trabajadores se sientan insatisfechos, haciéndoles caer dentro de un estado neutro dado que tampoco están satisfechos (Herzberg, 1968). De esa forma se evita la presencia de disgustos en los trabajadores.

Se resalta la necesidad de interpretar los resultados considerando las limitaciones de esta investigación. Una de las limitantes a considerar es la muestra por conglomerados utilizada dentro de esta investigación, por lo que los resultados deben ser analizados e interpretados con precaución.

\section{CONCLUSIONES Y RECOMENDACIONES.}

Fue a partir del análisis de la evidencia científica lo que permitió conocer los antecedentes teóricos que adquirieron el carácter de cimiento para orientar y sustentar la realización de esta investigación. Asimismo, con base en la percepción de los trabajadores mexicanos que se desempeñan en niveles de mandos medios y superiores (técnicos, coordinadores, supervisores y gerentes) y que pertenecen a una organización que se dedica a la manufactura de vestiduras automotrices, se construyó el modelo planteado, mismo que presentó altos índices de fiabilidad.

Como se predijo, la alienación laboral se relaciona negativa y significativamente con la satisfacción laboral, lo cual coincide con lo establecido a priori dentro de la evidencia científica disponible. Adicionalmente, la alienación laboral posee un efecto predictivo sobre la satisfacción laboral.

Otro aspecto que se debe de considerar es que la presente investigación identifica la litera científica actual que sustenta la vigencia de analizar a la alienación laboral desde un enfoque organizacional en el sector automotriz en Ciudad Juárez, Chihuahua. Es responsabilidad de las empresas dedicadas a la manufactura de vestiduras automotrices, el desarrollar actividades relativas a la proporción de mayor autonomía y de retroalimentación hacia el trabajador, mismas que otorguen un carácter más dinámico, haciendo que el entorno del trabajo sea más significativo e intrínsecamente satisfactorio para todos y cada uno de sus integrantes.

Se recomienda que para futuras investigaciones sería muy interesante replicar la evaluación de las variables en otros sectores productivos, como el médico y el de empaque por ejemplo. Asimismo, sería importante analizar la relación entre la alienación laboral y variables como el empoderamiento psicológico individual, cultura organizacional, la motivación per se y el compromiso organizacional. 


\section{Bibliografía}

Alonso, M. (2008). Estudio comparativo de la satisfacción laboral en el personal de administración. Revista de Psicología del Trabajo y de las Organizaciones, 2: 45-63.

Armstrong, M. (2004). The influence of prior commitment on the reactions of layoff survivors to organizational downsizing. Journal of Occupational Health Psychology, 9(1), 46-60.

Bacharach, S. y Aiken, M. (1979). The impact of alienation, meaninglessness, and meritocracy on supervisor and subordinate satisfaction. Social Forces, 57(3), 853-870.

Banai, M., Reisel, W. y Probst, T. (2004). A managerial and personal control model: predictions of work alienation and organizational commitment in Hungary. Journal of International Management, 10(3), 375-392

Bies, R. y Moag, J. (1986). Interactional justice: Communication criteria of fairness. Research on Negotiations in Organizations, 1, 43-55.

Blau, P. (1964). Exchange and power in social life. New York: Wiley.

Blauner, R. (1964). Alienation and freedom. Chicago: University of Chicago Press

Brender-Ilan, Y. (2012). How Do Income and Its Components and Perception Relate to Alienation?. Journal Of Applied Social Psychology, 42(2), 440-470.

Campbell, D. y Fiske, D. (1959). Convergent and discriminant validation by the multitrait-multimethod matrix. Psychological Bulletin, 56, 81-105

Ceylan, A. y Sulu, S. (2010). Work alienation as a mediator of the relationship of procedural injustice to job stress. South East European Journal of Economics and Business, 5(2), 65-74.

Chiaburu, D., Diaz, I., y de Vos, A. (2013). Employee alienation: relationships with careerism and career satisfaction, Journal of Managerial Psychology, 28 (1), 4 - 20

Chiaburu, D., Thundiyil, T. y Wang, J. (2014). Alienation and its correlates: A meta-analysis. European Management Journal, 32(1), 24-36

Chiang, V., Salazar, B. y Núñez, P. (2007). Clima organizacional y satisfacción laboral en un establecimiento de salud estatal: hospital tipo 1. Theoria, 16(2), 61-76.

Chin, W. (1998). Issues and Opinion on Structural Equation Modeling. MIS Quarterly, 22(1), 7-16.

Chin, W., Peterson, R. y Brown, S. (2008). Structural equation modeling in marketing: Some practical reminders. Journal of marketing theory and practice, 16(4), 287-298.

Cohen, J. (1992). A power primer. Psychological Bulletin, 112(1), 155-159

Cronbach, L. (1951). Coefficient alpha and the internal structure of tests. Psychometrika, 16, 1-16.

Dijkstra, T. y Henseler, J. (2015) Consistent partial least squares path modeling. MIS Quart. 39, 297-316.

Farrell, A. (2010). Insufficient discriminant validity: a comment on Bove, Pervan, Beatty and Shiu (2009). Journal of Business Research, 63(3), 324-327.

Fornell, C., y Larcker, D. F. (1981). Evaluating structural equation models with unobservable variables and measurement error. Journal of Marketing Research, 18, 39-50.

Galván, O. (2019). Efectos de la alienación laboral sobre la satisfacción laboral y el empoderamiento psicológico: Caso del sector automotriz de Ciudad Juárez, Chihuahua. (Tesis doctoral) Doctorado en Ciencias Administrativas, Universidad Autónoma de Ciudad Juárez, Ciudad Juárez, México.

García, R. y Fierro, E. (2019). Comportamiento desviado en el trabajo y compartir conocimiento: la relación y el efecto mediador de la alienación laboral. Pensamiento \& Gestión, (46), 108-136.

Garson, G. (2016). Partial least squares regression and structural equation models. Asheboro: Statistical Associates.

Gefen, D., y Straub, D. (2005). A practical guide to factorial validity using PLS-Graph: Tutorial and annotated example. Communications of the Association for Information Systems, 16(1), 91-109.

Gold, A., Malhotra, A. y Segars, A. (2001). Knowledge management: an organizational capabilities perspective.Journal of Management Information Systems, 18(1), 185-214 
Gotz, O., Liehr-Gobbers, K. y Kraff, M. (2010). Evaluation of Structural Equation Models Using the Partial Least Squares (PLS) Approach. En: V. Vinzi, W. Chin, J. Gouldner, A. (1960). The Norm of Reciprocity: A Preliminary Statement. American Sociological Review, 25: 161-178.

Greenberg, J.y Scott, K. (1996). Why do employees bite the hand that feeds them? Employee theft as a social exchange process. Research in Organizational Behavior 18: 111-155.

Hair Jr., J., Hult, G., Ringle, C. y Sarstedt, M. (2016). A primer on partial least squares structural equation modeling (PLS-SEM): Sage Publications, Inc.

Hair, J., Black, B., Babin, B., Anderson, R. y Tatham, R. (2005). Multivariate data analysis. New Jersey: Prentice-Hall.

Hair, J., Black, W., Babin, B., y Anderson, R. (2010). Multivariate data analysis. Englewood Cliffs: Prentice Hall.

Hair, J., Sarstedt, M., Pieper, T y Ringle, C. (2012). The use of partial least squares structural equation modeling in strategic management research: a review of past practices and recommendations for future applications. Long range planning, 45(5-6), 320-340.

Hegney, D., Plank, A. y Parker, V. (2006). Extrinsic and intrinsic work values: Their impact on job satisfaction in nursing. Journal of Nursing Management, 14, 271-281.

Henseler, J. (2017). Partial least squares path modeling. in: Leeflang, P.S., Wieringa, J.E.Bijmolt, T.H \& Pauwels, K.H. (Eds.), 361-382, Advanced Methods for Modeling Markets,Springer.

Henseler, J., Ringle, C. y Sarstedt, M. (2015). A new criterion for assessing discriminant validity in variance-based structural equation modeling. Journal of the Academy of Marketing Science, 43(1), 115-135.

Henseler, J., Ringle, C. y Sinkovics, R. (2009). The use of partial least squares path modeling in international marketing. Advances in International Marketing, 20, 277-320.

Herzberg, F. (1968). One more time: How do you motivate employees?. Harvard Business Review. Enero-Febrero, 53-62.

Hoppock, R. (1935). Job Satisfaction. New York: Harper and Brothers.

Hsu, S., Chen, W.y Hsieh, M. (2006). Robustness Testing of PLS, LISREL, EQS and ANN-based SEM for Measuring Customer Satisfaction. Total Quality Management, 17(3), 355-371.

Imran, M., Aziz, A. y Hamid, S. (2017). Determinants of SME export performance. International Journal of Data and Network Science, 1(2), 39-58.

INDEX (2019a). Información general IMMEX. Información Estadística. Recuperado de: https://indexjuarez.com/ wp-content/uploads/2019/07/Julio-5.pdf

INDEX (2019b). Información general IMMEX. Información Estadística. Recuperado de: https://indexjuarez.com/ wp-content/uploads/2019/05/24-Mayo.pdf

INDEX (2019c). Información general IMMEX. Información Estadística. Recuperado de: https://indexjuarez.com/ wp-content/uploads/2019/08/Julio-26.pdf

Islam, M., Mohammad, N. y Ahmed, A. (2013). A study on job satisfaction: focus on bankers of Bangladesh. European Journal of Business and Management, 5(17), 14-20.

Izquierdo, B. (2012). La obesidad y programas de atención en los estudiantes del área económico administrativo de diferentes universidades públicas. México. Investigación Administrativa, (109), 46-61.

Jiménez, A., Jara, M. y Miranda, E. (2012). Burnout, apoyo social y satisfacción laboral en docentes. Psicologia Escolar e Educacional, 16 (1), 125-134.

Kanungo, R. (1979). The concepts of alienation and involvement revisited. Psychological bulletin, 86(1), 119-138.

Kanungo, R. (1982), Work Alienation, New York: Praeger.

Kurdi, F. (2018). The Impact of Job Alienation on the Performance among Palestine Medical Complex Employees. International Humanities Studies, 5(3), 15-29.

Kwong-Kay, K. (2013). Partial least squares structural equation modeling (PLS-SEM) techniques using SmartPLS. Marketing Bulletin, Technical note1(24), 1-32. 
Levy, J., Varela, J. y Abad, J. (2006). Modelización con estructuras de covarianzas en Ciencias Sociales, Netbiblo: Madrid, España.

Marshall, G., Michaels, C. y Mulki, J. (2007). Workplace Isolation: Exploring the Construct and IRS Measurement. Psychology y Marketing, 24, 195-223.

Martín, P., Salanova, M. y Peiró, J. (2003). El estrés laboral: ¿Un concepto cajón-desastre?. Proyecto Social,10 (11), 167-186.

Martínez, M.y Fierro, E. (2018). Aplicación de la técnica PLS-SEM en la gestión del conocimiento: un enfoque técnico práctico. Revista Iberoamericana para la Investigación y el Desarrollo Educativo, 8(16), 130-164.

McKinlay, J. y Marceau, L. (2011). New wine in an old bottle: Does alienation provide an explanation of the origins of physician discontent? International Journal of Health Services, 41(2), 301-335

Meliá, J. y Peiró, J. (1989). La medida de la satisfacción laboral en contextos organizacionales: el cuestionario de satisfacción S20/23. Psicologemas, 3, 59-74.

Moch, M. (1980). Job involvement, internal motivation, and employees' integration into networks of work relationships. Organizational Behavior and Human Performance, 25, 15-31.

Montgomery, C. y Runger, G. (2008). Estatística aplicada e probabilidade para engenheiros. São Paulo: LTC.

Mottaz, C. (1981). Some Determinants of Work Alienation. Sociological Quarterly, 22(4), 515-529.

Nair, N. y Vohra, N. (2009). Developing a new measure of work alienation. Journal of Workplace Rights, 14(3), 293-309.

Nasurdin, A., Ramayah, T. y Kumaresan, S. (2005). Organizational Stressors and Job Stress among Managers: The Moderating Role of Neuroticism. Singapore Management Review 27 (2): 63-79.

Nunnally, J. y Bernstein, I. (1994). Psychometric Theory. New York: McGraw-Hill.

Özer, Ö., Uğurluoğlu, Ö., Saygilı, M. y Sonğur, C. (2019). The impact of work alienation on organizational health: A field study in health sector. International Journal of Healthcare Management, 12(1), 18-24. TURQUIA

Peiró, J. (2001). El estrés laboral: una perspectiva laboral y colectiva. Prevención, Trabajo y Salud, 13, 1-12.

Quero, M. (2010). Confiabilidad y coeficiente Alpha de Cronbach. Telos, 12(2), 248-252.

Quevedo, H., García, H., Salas, J., Domínguez, A. y Esquivel, V. (2007). Análisis estadístico de ozono a nivel del suelo. CULCyT, 4(21), 35-43.

Reinartz, W., Haenlein, M. y Henseler, J. (2009). An empirical comparison of the efficacy of covariance\#based and variance\# based SEM. International Journal of Market Research, 26, 332-344.

Ringle, C., Wende, S. y Becker, J. (2015). SmartPLS 3. Boenningstedt: SmartPLS GmbH.

Salgado, A. (29 de Mayo de 2019). Van maquiladoras vs estrés y acoso laboral. El Diario. Recuperado de: https:// diario.mx/juarez/van-maquiladoras-vs-estres-y-acoso-laboral-20190528-1521038/

Sarros, J., Tanewski, G., Winter, R., Santora, J. y Densten, I. (2002). Work Alienation and Organizational Leadership. British Journal Of Management, 13(4), 285-304.

Schultz, D. y Schultz, S. (1990). Psychology and Industry Today: An Introduction to Industrial and Organizational Psychology, New York: McMillan Publishing.

Seeman, M. (1959). On The Meaning of Alienation. American Sociological Review, 24(6), 783-791.

Seidel, G. y Back, A. (2009). Success factor validation for global ERP programmes. Paper presented at the 17th European Conference of Information Systems. ECIS, Verona, Italia.

Seybolt, J., y Gruenfeld, L. (1976). The discriminant validity of work alienation and work satisfaction measures. Journal OfOccupational Psychology, 49(4), 193-202.

Shepard, J. (1970). Functional specialization, alienation, and job satisfaction. ILR Review, 23(2), 207-219.

Shyu, C., Li, Y. y Tang, Y. (2013). Applying confirmatory factor analysis on the measure for restaurant over-service. The Journal of International Management Studies, 8(2), 10-16.

Singh, P. y Loncar, L. (2010) Pay satisfaction, job satisfaction and turnover intent, Relations Industrielles/Industrial Relations, 65 (3), 470-490. 
ReCai Revista de Estudios en Contaduría, Administración e Informática, 019, 8(23), Septiembre-Dic...

Sirin, E., Duman, S. y Karakus, S. (2011). Work alienation in predicting job satisfaction among physical education teachers in Turkey. World Applied Sci.J., 12, 1207-1213

Siron, R., Muttar, A., Ahmad, Z. y Tarsipan, M. (2016). The Mediating Role of Job Satisfaction on the Relationship between work alienation and job performance. International Business Management, 10(16), 3598-3606.

Triola, M. (2009) Estadística. México: Pearson Educación.

Vroom, V. (1964). Work and motivation. New York: Wiley

Werst, C., Linn, R. y Jöreskog, K. (1974). Interclass Reliability Estimates: Testing Structural Assumptions. Educational and Psychological Measurement, 34, 25-33.

Wold, S., Esbensen, K. y Geladi, P. (1987) Principal Component Analysis. Chemometrics Intelligent Laboratory System, 2, 37-52.

Wu, M. (2008). Structural Equation Modeling-Amos Operation and Application. Taipei: Wu Nan.

Zoghbi, P. y Caamaño, G. (2010). El Impacto de la Alienación Laboral sobre las Conductas Desviadas en el Trabajo: Un Estudio Exploratorio. Revista de Psicología del Trabajo y de las Organizaciones, 26(1), 79-92.

Copyright 2019 Universidad Autónoma del Estado de México

CC BY-NC-ND 